\title{
A Framework for Characterizing the Wireless and Mobile Network Continuum.
}

\author{
Yang Chen, Vincent Borrelł Mostafa Ammar, Ellen Zegura \\ School of Computer Science, Georgia Institute of Technology \\ Atlanta, GA, USA \\ yangchen@cc.gatech.edu, ammar@cc.gatech.edu, ewz@cc.gatech.edu, \\ vinouz@yahoo.fr
}

\begin{abstract}
The vast majority of research in wireless and mobile (WAM) networking falls in the MANET (Mobile Ad Hoc Network) category, where end-to-end paths are the norm. More recently, research has focused on a different Disruption Tolerant Network (DTN) paradigm, where end-to-end paths are the exception and intermediate nodes may store data while waiting for transfer opportunities towards the destination. Protocols developed for MANETs are generally not appropriate for DTNs and vice versa, since the connectivity assumptions are so different. We make the simple but powerful observation that MANETs and DTNs fit into a continuum that generalizes these two previously distinct categories. In this paper, building on this observation, we develop a WAM continuum framework that goes further to scope the entire space of Wireless and Mobile networks so that a network can be characterized by its position in this continuum. Certain network equivalence classes can be defined over subsets of this WAM continuum. We instantiate our framework that allows network connectivity classification and show how that classification relates to routing. We illustrate our approach by applying it to networks described by traces and by mobility models. We also outline how our framework can be used to guide network design and operation.
\end{abstract}

Categories and Subject Descriptors: C.2.1[Network Architecture and Design]: Wireless Networks

General Terms: Design

Keywords: Wireless and Mobile Networks, Mobile AdHoc Networks, Disruption-Tolerant Networks

\section{INTRODUCTION}

Wireless data networks with mobile nodes have been the subject of extensive research for at least three decades. Early efforts focused on networks frequently called Mobile Ad Hoc Networks (MANETs). While the nodes in such networks are mobile, it is generally assumed that end-to-end, multi-hop paths between node pairs exist most of the time. That is, end-to-end connectivity is the norm. Routing protocols designed to operate in MANETs assume that these paths are formed by a set of wireless links that exist contemporaneously [2,3]. If MANET paths are disrupted because of node

*This work was supported in part by NSF Grant NETS0519784 and CNS-0831714.

${ }^{\dagger}$ Work done while this author was a PhD student at LIP6 (Paris, France) and visiting the School of Computer Science at Georgia Tech. mobility, then this disruption is only temporary, and identical or alternate paths are restored relatively quickly.

Recent research has focused on a different paradigm for wireless and mobile networks. This paradigm goes by various names: disruption-tolerant, delay-tolerant, opportunistic or intermittently-connected networks $[4,5,6]$. We will use the "DTN" acronym to refer to this class of networks. DTNs differ from MANETs in end-to-end connectivity. In a DTN, the links on an end-to-end path may not exist contemporaneously and intermediate nodes may need to store data waiting for opportunities to transfer data towards its destination. That is, end-to-end connectivity is the exception. We call end-to-end DTN paths space-time paths to distinguish them from contemporaneous space paths used in MANETs [7].

For any particular network, the question of whether it is a MANET or a DTN (or equivalently a network with space paths or space-time paths) is critical to answer. This question has generated some interesting discussion within the DTN community ${ }^{1}$. Protocols developed for MANETs generally do not work in DTNs and vice versa, since the connectivity assumptions are so different; hence categorizing a network is critical to its effective operation. In practice, the question of network category is challenging to formulate and answer. Many networks will not fit neatly within a simple classification scheme and/or they may change their classification over time. Informally, how a network is classified depends on network features such as the size of the network, the geographical area covered by the network, and the node mobility pattern. Additional factors, such as traffic pattern and application requirements, also often affect how a network is classified. Except for some extreme cases, it is generally not a priori obvious as to which class a particular network belongs.

This paper aims to take a fresh perspective on classifying wireless and mobile networks (WAMs) networks. We develop a framework which provides a unified treatment of wireless and mobile networks. Our main premise is that it is best to consider the space of $\mathrm{WAMs}^{2}$ as a continuum. A particular network is characterized by its position in this continuum, which may change over time. Our characteri-

\footnotetext{
${ }^{1}$ See http://maillists.intel-research.net/pipermail/dtninterest/2010-January/thread.html

${ }^{2}$ We use the term WAM to describe all wireless and mobile networks to emphasize the fact that we include all classes of such networks in our terminology and not ones that have any specific properties.
} 
zation can apply to an entire network or can be different for different parts of the network (e.g., different node pairs). Certain network equivalence classes can be defined over subsets of this WAM continuum and such network classification can be used to help guide WAM design and operation.

The unified view provided by our framework formalizes the widely held informal notion that wireless and mobile network classes such as MANETs and DTNs that have often received distinct treatment in the literature are "related". Additionally it provides a framework for understanding networks that either don't fit neatly into one category or can change classification over time. It can also form the core of a model of operation in which networks can adapt over time based on changes in their position in the WAM continuum and it can provide insights into network performance.

Our framework provides the means by which one can describe connectivity properties in a WAM. In networks where data is routed on contemporaneous end-to-end paths, it is simple to discuss connectivity properties. The network (or more specifically a node pair) is either connected or not. In DTNs, the concept of space-time path connectivity is more complex and needs to incorporate notions of connectivity over time. As a result, the problem of developing a unifying framework for WAMs presents many challenges, including developing a formalism for specifying network classes, incorporating parameters that reflect some level of abstraction of protocols within the classification framework, and developing procedures and algorithms for performing classification based on network and protocol parameters and features. We tackle each of these challenges in this paper.

In this paper we present a specific instantiation of our framework which provides guidance on what type of routing protocol is feasible for WAM networks. The goal is to have the classification be usable to determine the most appropriate routing strategy for a network. Unlike our earlier work on this topic [8], this new work admits granularity at the level of node-pairs, thus accommodating classification of portions of the network as well as the network as a whole.

We illustrate our approach by applying it to networks described by traces from real platforms and mobility models. Our results aim to demonstrate the power of our unifying approach in providing significant insight into the effect of network parameters and features on network character and performance. They also show how instances of our framework can be used as a systematic and formal descriptive and evaluative tool.

The rest of this paper is structured as follows. In Section 2 we provide an overview of our framework and its basic concepts. In Section 3 we describe our network model and our use of an evolving graph to represent WAMs. In section 4, we develop and illustrate an instantiation of our framework that provides a formal node pair connectivity classification. The node-pair classification is used in Section 5 as the basis for a whole network classification exercise which is also illustrated through examples. Section 6 outlines some possible uses of our framework in WAM network design and operation scenarios. Section 7 concludes this paper.

Additional results and extensions are reported in a longversion of this paper available on-line [1].

\section{THE WAM CONTINUUM FRAMEWORK}

\subsection{Overview}

We consider wireless and mobile networks (WAMs) in which nodes equipped with wireless interfaces can move within a given space. Nodes establish contacts with other nodes that are within their radio range. For our purposes, a WAM is fully specified by the contacts between its nodes as well as properties of each contact (e.g., duration and data rate).

In general, all nodes may be sources and destinations for data. In addition, nodes can act as intermediate nodes on the path from a source to a destination of data. In some cases, an intermediate node will store data temporarily until it is capable of forwarding data towards the destination.

In general, data may be forwarded along a space-time path $^{3}$ in an evolving graph[11]. A space-time path is one in which the links may not exist contemporaneously. Data is forwarded by being transmitted on a link in the path when the link exists and then being stored at the intermediate node awaiting the formation of the next link. Note that a space path with contemporaneous links is simply a special case of a space-time path.

We envision that each fully specified WAM represents a point in a multi-dimensional space that we call the WAM continuum. To illustrate the concept, consider the definition of a two-dimensional space that uses the average node density and the average node speed as the two dimensions. A given WAM network will occupy a point in this two dimensional space based on its density and speed properties. We can group points on a WAM continuum into classes such that networks within a specific class possess equivalent properties. Consider, for example, the following informal classification of our two-dimensional WAM continuum into three classes: Class 1: networks with reasonably persistent space paths (high density and low speed), Class 2: networks with no paths (low density and low speed) and Class 3: networks where node-pairs are connected with paths that form over time (space-time paths). This is illustrated informally in Figure 1. We will develop our classification more formally in the next section.

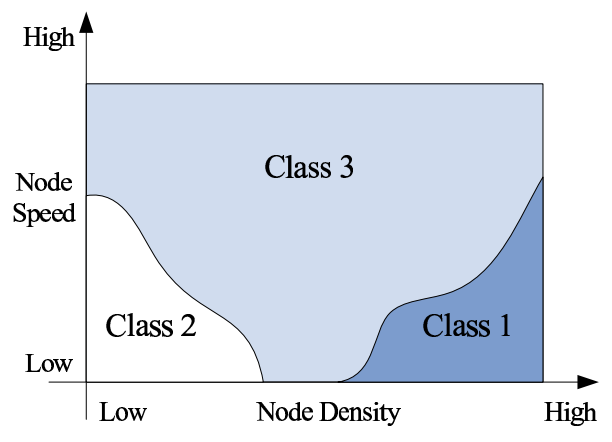

Figure 1: WAM Continuum Classification Example

\subsection{Basic Concepts}

There are several important concepts to keep in mind as we further develop the WAM continuum framework.

- The WAM Continuum admits many variations. In principle, one can possibly talk about a unique WAM continuum

\footnotetext{
${ }^{3}$ Space-time paths are similar to definitions in $[7,9,10]$.
} 
in which all WAMs can be placed. However, this general space will have a large number of dimensions and will be very difficult to define. Consequently, and for practical reasons, our instantiation of the framework will have a small number of dimensions.

- The WAM continuum can have different time and space granularity. The informal example shown above considers the placement and classification of entire networks over the complete network lifetime. Our framework allows for a finer granularity that considers spatial parts of networks or different classifications over time. In the specific instantiation we develop next, we start with a taxonomy for node-pair connectivity which is ultimately built up into definitions for a classification of entire networks.

- A network's (or a node-pair's) place in a WAM continuum should depend on intrinsic network properties and not on protocols or procedures we choose to use in data delivery. In our discussion of WAMs we will provide a clear distinction between our description of basic network properties and our description of protocols which we use to deliver data over the network. While this might seem obvious, it does bear emphasizing especially because networking researchers frequently do not make such a distinction in terminology.

- The WAM continuum is not naturally divided into classes. While it is convenient to talk about network or node-pair classes, we view the WAM space as a continuum without any "natural" boundaries. We will find that imposing a discrete classification is useful for us to get a handle on complexity. However, we will also find that such classification can be quite flexible. Our goal will, therefore, be to provide classification instances that can be augmented or enhanced as may be required by additional considerations.

\section{WAM NETWORK MODEL}

We consider wireless and mobile networks comprising a number of nodes equipped with wireless interfaces moving within a given space. Communication between two nodes is established when they are within radio range of each other. Data can be exchanged between nodes during those contact periods. Therefore, in terms of the data transfer opportunities, a WAM is fully specified by the sequence of contacts between its nodes.

At any given time, a graph $G=(V, E)$ can be used to describe nodes and links. In order to accurately describe a general WAM with time-varying contacts, we use the model based on the concept of evolving graphs [11].

DeFINITION 1. Evolving Graph [11]: An evolving graph $\mathcal{G}=\left(G, S_{G}, S_{T}\right)$ comprises a graph $G=(V, E)$, the sequence of its $\mathcal{T}$ subgraphs $S_{G}=G_{1}, G_{2}, \ldots, G_{\mathcal{T}}$ and the sequence of its $\mathcal{T}+1$ time instants $S_{T}=t_{0}, t_{1}, t_{2}, \ldots t_{\mathcal{T}} . G_{k}$ is the subgraph in place during $\left[t_{k-1}, t_{k}\right)$.

It is relatively straightforward to see how a wireless and mobile network can be described as an evolving graph. As nodes move they potentially acquire and shed neighbors, changing the shape of a graph. The exact nature of these neighbor changes is a function of the node mobility and can be captured by the specifics of graph evolution.

Our notion of space-time paths is captured by the definition of journeys as follows:

Definition 2. Journey [11]: A journey $\mathcal{J}=\left(R, R_{\delta}\right)$ in an evolving graph $\mathcal{G}$ is comprised of $R=e_{1}, e_{2}, \ldots, e_{k}$ the sequence of edges it traverses, and $R_{\delta}=\delta_{1}, \delta_{2}, \ldots, \delta_{k}$ the corresponding time instants of edge traversal. $R_{\delta}$ must be such that each edge traversed is in the evolving graph at the time of traversal.

We use the terms "space-time path" and "journey" interchangeably in this paper.

\section{NODE PAIR CLASSIFICATION}

\subsection{An Idealized Node Pair Classification}

For the following definitions we assume that an evolving graph $\mathcal{G}$ describes the WAM under consideration.

Definition 3. Space-Path Pair (S-Pair): A pair of nodes $i$ and $j$ is called an $S$-Pair if a space path can always be found between them in $\mathcal{G}$.

Data can still be transferred between node pairs which are not S-Pairs using store-carry-forward routing. To capture the character of those node pairs, we have the following node pair definition:

Definition 4. Space-Time-Path Pair (ST-Pair): A pair of nodes $i$ and $j$ is an ST-Pair if: $\forall t$, a journey exists in $\mathcal{G}$ between $i$ and $j$ at $t$, and this pair is not an $S$-Pair.

It is possible that no path can be found between particular node pairs after certain time point. Therefore, we introduce the last node pair class.

Definition 5. No-Path Pair (N-Pair): A pair of nodes $i$ and $j$ is an $N$-Pair if $\exists t$ such that no journey can be found from $i$ to $j$ after $t$ in $\mathcal{G}$.

Finally, it is important to note that both the ST-Pair and the N-Pair definitions are not symmetric. That is if nodepair $(i, j)$ is either an ST-pair or an N-Pair then node $(j, i)$ might follow a different classification.

\subsection{A Practical Classification}

We now augment our node-pair classification above to incorporate some degree of practical routing consideration. To understand the motivation, consider a network where connectivity changes rapidly but where a particular node pair is always connected through a space path. While a space path exists all the time, the actual links forming the path change in such a manner that no specific path persists for a long time. According to our definitions above, this pair will be classified as an S-Pair and one may reach the conclusion that a MANET routing protocol may be suitable for routing between the two nodes. However, such routing protocols need some time to discover a route and therefore require a certain amount of route persistence. In that case we need to modify our S-Pair definition to take this into account. To that end we define space path persistence as follows:

Definition 6. Space Path Persistence Time: Let $i$ and $j$ be an $S$-Pair in an evolving graph $\mathcal{G}$. By definition, the node pair is always connected by a space path that may change over time. We denote the sequence of space paths connecting the two nodes over time as $p_{1}, p_{2}, p_{3}, \ldots$ The persistence time for path $p_{i}$ is the time from when it is first formed in the network until the path no longer exists due to node mobility and is replaced by a new path $p_{i+1}$. 
Assuming that routing protocols need at least $\delta$ time units to discover a new path, we define the following:

Definition 7. $(\delta)$ S-Pair: $A(\delta) S$-Pair is an S-Pair where all the paths have persistence time greater than or equal to $\delta$.

Now consider node pairs classified as ST-Pair. For such pairs, the important consideration is the duration of the journey providing the space-time path. While such journeys are usable if some form of DTN routing is used, such networks often impose a lifetime beyond which data is not usable. This leads to the following definition.

Definition 8. $(\gamma)$ ST-Pair: A $(\gamma)$ ST-Pair is an STpair where the journeys according to Definition 4 are always less than or equal to $\gamma$ in duration.

\subsection{Classifying Node Pairs Over Time}

The previous classification provides a rather strict categorization of node pairs. For example, a node-pair that is connected through a space path but loses its connectivity even for a brief period of time is not classified as an SPair. To address this issue we propose to classify network over time. Time is broken up into epochs during which the network connectivity (or graph in an evolving graph) does not change. Our goal is to provide a classification for each node pair in each epoch according to the following criteria for given $\delta$ and $\gamma$ :

- A node-pair is classified as an S-Pair during epoch $k$ represented by subgraph $G_{k}$ (one of the series of subgraphs in the evolving graph) if the node pair is in the same connected component of $G_{k}$ and either the epoch lasts for at least $\delta$ or the path connecting the node pair does not change for at least $\delta$ time units and this period consists of one or multiple epochs including epoch $k$.

- A node-pair $(i, j)$ is classified as an ST-Pair during epoch $k$ if it is not classified as an S-Pair in this epoch and one can find a journey from $i$ to $j$ of duration no more than $\gamma$ starting form the beginning of this epoch.

- A node-pair is classified as an N-Pair otherwise.

\subsection{Examples of Node Pair Classification}

We now illustrate our node-pair classification methodology by applying it to networks represented by two types of contact sequences: traces collected from real WAM deployment or experiments [12] and synthesized ones from Random Way Point (RWP) mobility model. we consider the latter for its simplicity in tuning different parameters to highlight the interesting capability of our classification scheme. It should be clear that our goal in this illustration is to demonstrate the descriptive and evaluative power of our framework and not to compare various network operation strategies.

With $\delta=5$ seconds and $\gamma=3600$ seconds, we first show epoch by epoch node pair classification of a 9 node trace [13] collected in the Haggle Project [12] in Figure 2. Time is on the $\mathrm{x}$-axis and the 72 node-pairs are represented on the $\mathrm{y}$ axis. Different node pair classes are represented by different shades as indicated by the legend. Note that each node pair appears twice on the $\mathrm{x}$-axis but in a reverse direction.

The insight into properties of the trace achievable from this visualization of the outcome of our classification is evident. For example, the long periods of graph disconnection during which nodes are mostly disconnected is apparent.

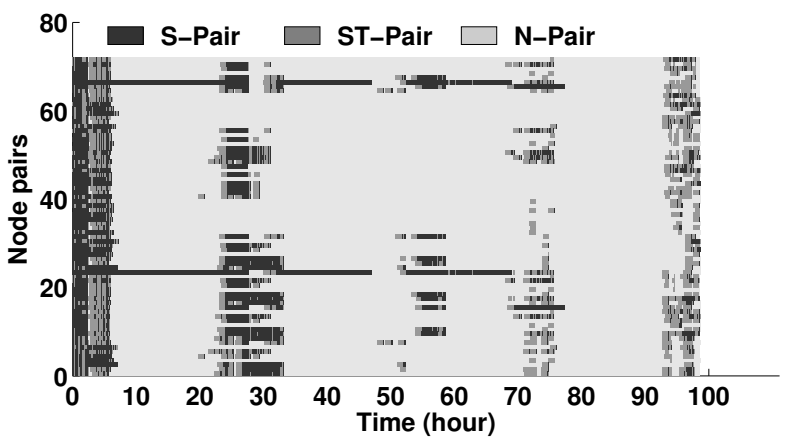

Figure 2: Epoch by Epoch Node Pair Classification

The interval of network characteristic changes follows human daily activity cycles, i.e., 24 hours. The fact that two nodes are in almost constant contact is represented by the two black horizontal lines (actually representing the same node pair).

We also show the percentage of time that each node pair belongs to each classification in Figure 3(a). Note that overall the classification varies significantly among all node pairs, which indicates the spatially heterogeneous character of this WAM network.

The impact of the parameters $\gamma$ on the classification of node pairs in the Haggle trace is illustrated in Figure 3(b). A larger $\gamma$ value presents a more relaxed constraint on the allowed journey duration. A node pair classified as an NPair in some epochs may be reclassified as ST-Pair. We notice an increase in the ST-Pair percentage on most node pairs in Figure 3(b) when $\gamma$ increases from 1 hour to 8 hours.

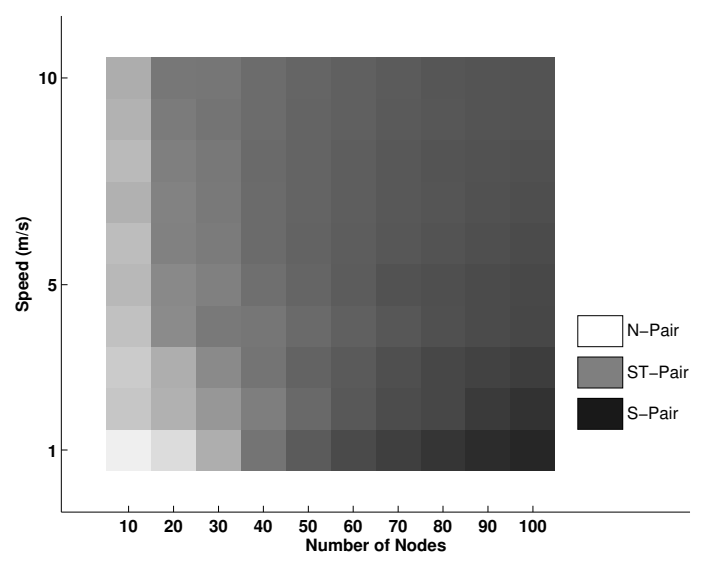

Figure 4: Node Pair Classification on RWP Trace

We next show classification results using synthetic traces derived from the Random Way Point model. In our model a given number of nodes move in a $2 \mathrm{~km}$ by $2 \mathrm{~km}$ area for 3 hours. Their communication range is $250 \mathrm{~m}$. The pausing time is uniformly distributed between 0 and 10 seconds. We adjust the number of nodes and the average node speeds in our experiments.

Because all nodes (and hence all node pairs) are homogeneous in our model, we focus on the classification of a specific node pair chosen at random. Our goal is to show how the node pair classification changes as network and mo- 


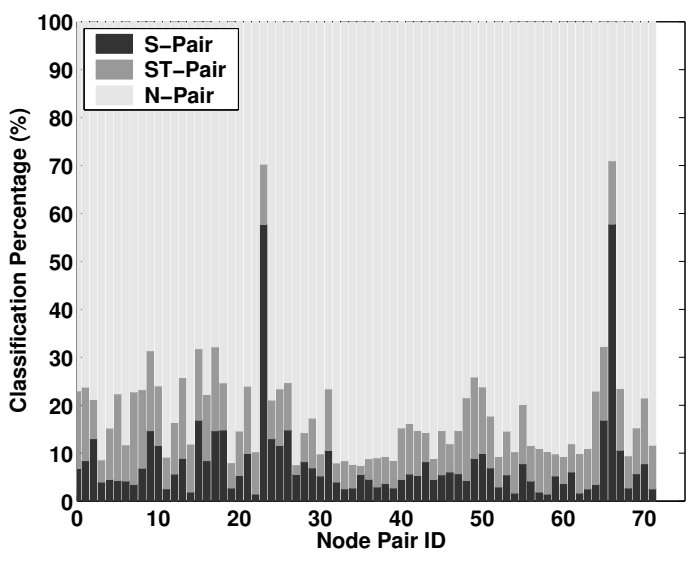

(a) $\gamma=1$ hour, $\delta=5$ seconds

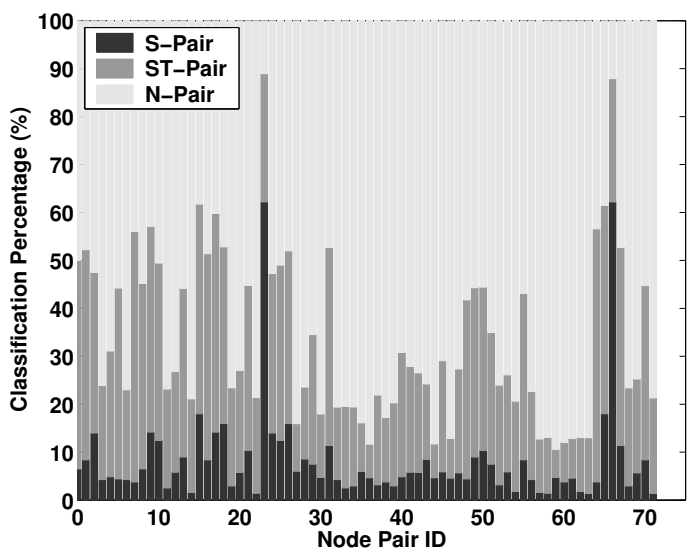

(b) $\gamma=8$ hour, $\delta=5$ seconds

Figure 3: Node Pair Classification of the Haggle Trace

bility parameters change. The results are shown in Figure 4. Each square in the space represents the chosen node pair in a network with the corresponding number of nodes ( $\mathrm{x}$-axis) and average speed (y-axis). Three colors (white, gray and black) are used to represent node pair classes (N-Pair, STPair and S-Pair). Each square is filled in with a mixture of these colors that represents the percentage of time the node pair spends in each class. The reader should compare this figure with the one sketched in Figure 1.

Figure 4 shows how network features affect the character of node pairs. When node density (number of nodes) is low, increasing speed increases the chance that a space time path is found between node pairs. When node density is high, most of the nodes are connected. However, a lower average speed keeps the (possibly multi-hop) connectivity between the node pair more stable and thus more time it is classified as S-Pair.

\section{WHOLE NETWORK CLASSIFICATION}

We now show how the per-node-pair classification described above can be aggregated to classify whole networks. In [8], three categories of network are proposed: Space Path Network (SPN) in which MANET routing protocols can be applied among all nodes, Unassisted-DTN (U-DTN) in which DTN routing protocols (such as epidemic routing [4] or probabilistic routing [6]) are able to deliver data between all node pairs; and Assistance-needed-DTN (A-DTN) in which extra assistance (such as message ferrying [15]) is needed because no space/space-time path can be found between some node pairs.

While we use similar terminology for whole network classification as [8], the approach in this paper is different. The previous work develops an "all-or-nothing" whole network classification, whereas the more general approach developed here builds network classification bottom-up from node-pair classification components. In addition, we allow some flexibility in the definition where a network classification can be inherited from a classification of a proportion of node-pair classification. This is formalized in the definitions below.
DEFINITION 9. $x-S P N$ : A network is classified as an $x$ $S P N$ in an epoch if at least $x \%$ of the node pairs are classified as $S$-Pairs during that epoch.

Definition 10. $x$-U-DTN: A network is classified as an $x-U-D T N$ in an epoch if at least $x \%$ of the node pairs are classified as either S-Pairs or ST-Pairs and the network is not classified as $x-S P N$ during that epoch.

Definition 11. $x-A-D T N$ : A network is classified as an $x-A-D T N$ in an epoch if it is neither an $x-S P N$ nor an $x$ $U-D T N$.

Similarly as in node pair classification, in order to capture the potential network character change over time, we classify the network in each epoch and the network classification over its lifetime is given by the percentage of time that it spends in each class ${ }^{4}$.

\subsection{Examples of Network Classification}

\begin{tabular}{|c||c|c|c|}
\hline $\mathrm{X}$ & $\mathrm{x}-\mathrm{SPN}$ & $\mathrm{x}-\mathrm{U}-\mathrm{DTN}$ & $\mathrm{x}-\mathrm{A}-\mathrm{DTN}$ \\
\hline 100 & $0.5 \%$ & $6.6 \%$ & $92.9 \%$ \\
\hline 90 & $0.5 \%$ & $7.7 \%$ & $91.8 \%$ \\
\hline 50 & $3.3 \%$ & $17.3 \%$ & $79.4 \%$ \\
\hline
\end{tabular}

Table 1: Network Classification on the Haggle Trace

We first apply our classification method to the Haggle trace with $\delta=5$ seconds and $\gamma=3600$ seconds. The results are presented in Table 1 . When $x=100$, the network is mostly classified A-DTN due to the strictness of the $100 \%$ constraint. As $x$ decreases, classification results show that a significant number of space and space-time paths actually exist for certain epochs, thus revealing an intrinsic property of the network.

\footnotetext{
${ }^{4}$ Note that while the classification in [8] is closely related to the classification presented here when $x=100 \%$, it is not identical because of the manner in which we define whole network classification as an aggregation of node-pair classification.
} 


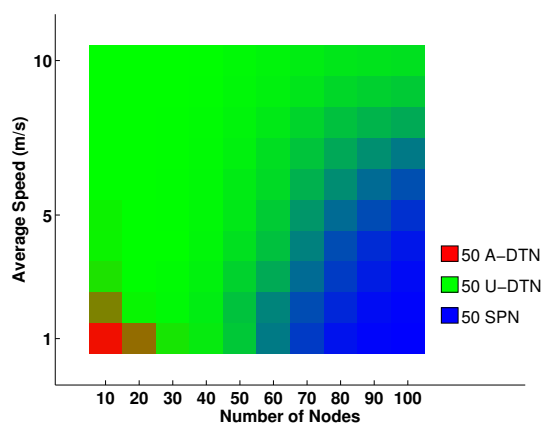

(a) $\mathrm{x}=50 ; \gamma=600 ; \delta=5$

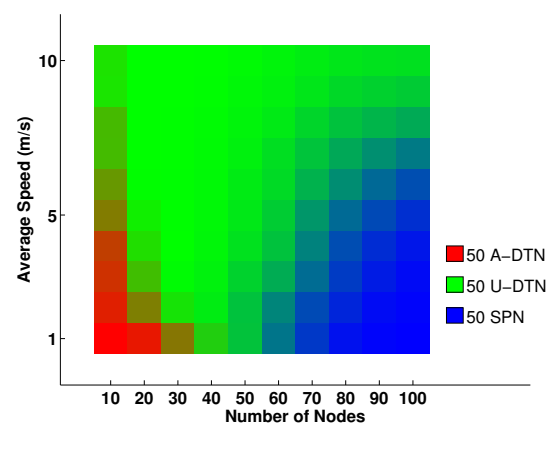

(b) $\mathrm{x}=50 ; \gamma=300 ; \delta=5$

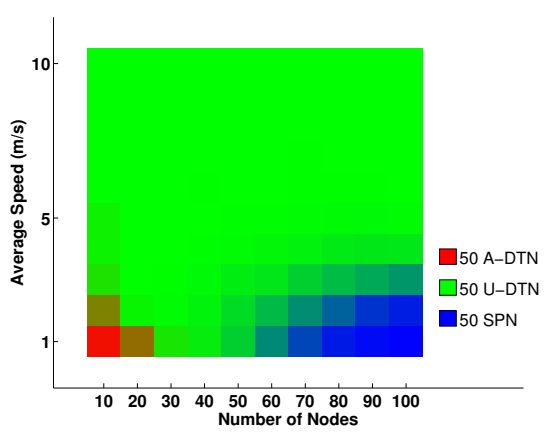

(c) $\mathrm{x}=50 ; \gamma=600 ; \delta=10$

Figure 5: Joint Effect of Node Density and Speed on Network Classification: Traces from RWP Model

The table shows the flexibility of our framework to adjust the network's classification depending on customized objectives. The parameter $x$ allows us to specify when we consider the network connected. For example with $x=50$ we say that a network is connected (with space or spacetime) paths if at least $50 \%$ the node pairs are connected. The table shows that this is true for about $20 \%$ of the time (the sum of SPN and U-DTN connectivity percentages). It implies that for our more relaxed definition of connectivity the network will need assistance for around $80 \%$ of the time. The first row of the table shows that the network will need assistance for around $92 \%$ of the time if we adopt a more strict definition of connectivity $(x=100)$.

We next show classification results using synthetic traces derived from the Random Way Point model with the same setting used in the node pair classification study. We adjust the number of nodes and the average node speeds in our experiments. The results are shown in Figure 5. Each square in the space represents a network with the corresponding number of nodes (x-axis) and average speed (y-axis). Different colors represent network classes (A-DTN, U-DTN and A-DTN) now. Each square is filled in with a mixture of these colors that represents the percentage of time the network spends in each class. These figures should be compared to the informal sketch in Figure 1: the darker shades correspond to Class 1 networks and the lighter shades correspond to Class 3 networks.

These figures show that when node density (number of nodes) is low, increasing speed creates more space time paths. However, when node density is high, a high speed causes more unstable space paths and lower SPN classification accordingly. Similarly, when the node speed is high, although high node density means more connectivity, the space paths are less stable.

The effect of other parameters in the classification procedure is also shown in Figure 5. With a smaller $\gamma$ value, it is more difficult to find a space time path with a satisfying duration. The time percentage that the network is classified as A-DTN increases accordingly. On the other hand, a larger $\delta$ value puts a stricter constraint on space path persistence. Even with the same network features, i.e., number of nodes and average node speed, less "usable" space paths are found and the network is classified as SPN in less epochs.

\section{APPLYING THE WAM CONTINUUM FRAMEWORK}

In this section we outline how our framework may be applied to guide WAM design and operation. At a fundamental level, developing a classification of a particular network is an algorithmic technique that enables a characterization of the network. Once available, a network's classification and how it fits in a larger continuum can be useful in a number of ways.

\section{Predicting network performance.}

Applying our WAM continuum framework can be a relatively low-effort exercise (compared to simulation or detailed mathematical analysis) and can often provide important first insights into network performance. These insights can be used to quickly eliminate designs or flag performance issues. To illustrate this point we show an example where there is a strong correlation between a network's classification and its data delivery performance. In the example we use the haggle contact trace from Section $4.4[12,13]$ and run a simulation using the ONE simulator [14]. Simulation parameters are set as follows: TTL $=1$ hour; traffic load is $1 \mathrm{kbps}$ between each node pair with average message size of $100 \mathrm{~KB}$; the buffer size on each node is $50 \mathrm{MB}$ and probabilistic routing [6] is used. The correlation between node classification results and message delivery ratio is given in Figure 7 . The figure shows how classification can be used as a performance predictor. Ultimately successful performance prediction through classification will depend on a number of factors such as the traffic load on the network ${ }^{5}$. Future research should investigate the predictive power and limitations of the classification framework.

\section{Understanding the effect of design alternatives.}

There are often many alternatives in the design and operation of WAMs. Our classification framework can provide quick insights into the effect of various design or operation decisions. To illustrate this consider the question of whether or not to provide a message ferry[15]. In message ferrying, a special node called the ferry moves in the network space and provides data movement capability among nodes. We

\footnotetext{
${ }^{5}$ Heavily loaded networks perform poorly no matter what their classification is.
} 


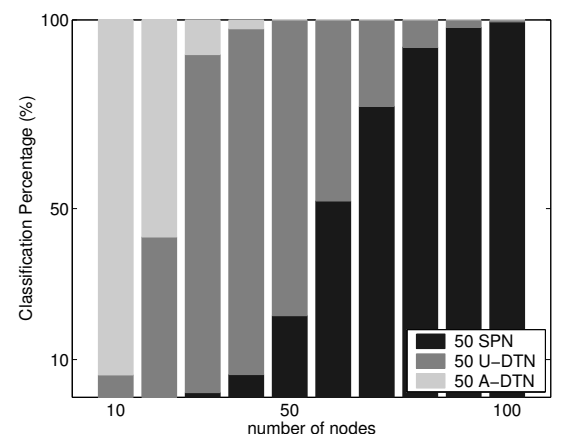

(a) With no Ferry

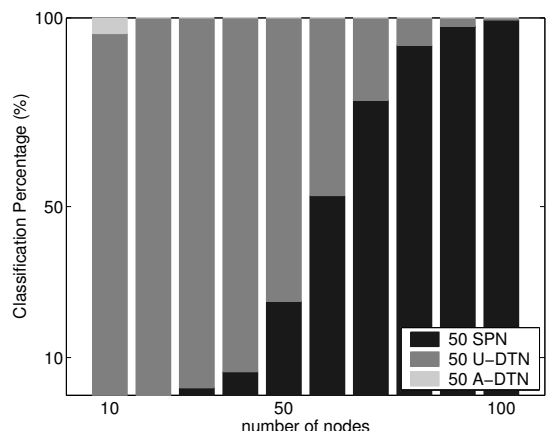

(b) With 1 Ferry

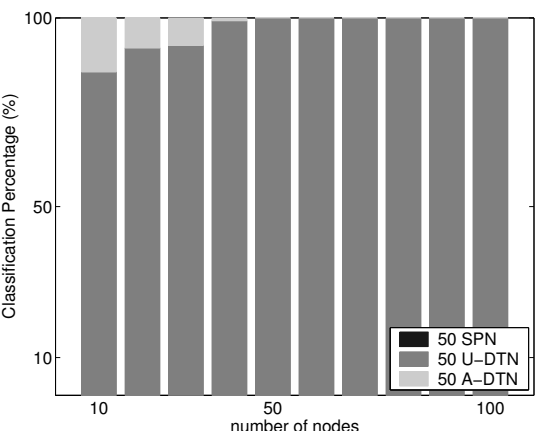

(c) Ferry/node Contacts only

Figure 6: Effect of Message Ferrying on Network Classification

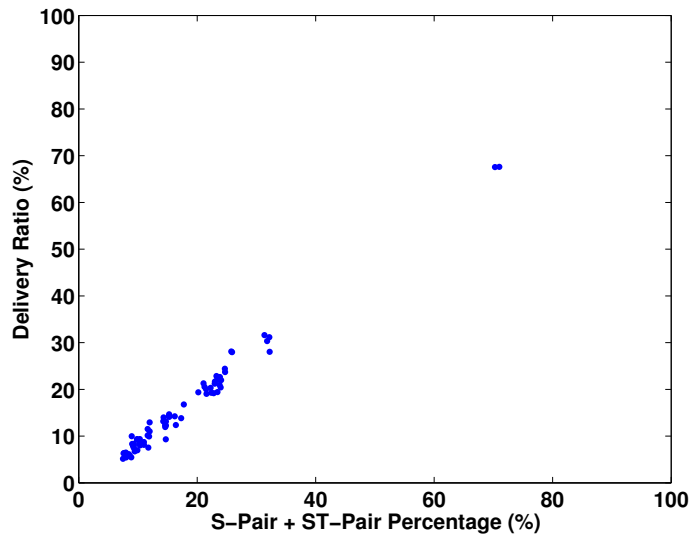

Figure 7: Correlation between Classification and Simulation Result

consider network classification using a trace generated from the RWP model described in Section 5 with average node speed of $1 \mathrm{~m} / \mathrm{s}$. We consider two scenarios: one with just the nodes and the other in which we introduce a message ferry moving along a square route with diagonal points $(500,500)$ and $(1500,1500)$ at $20 \mathrm{~m} / \mathrm{s}$. Figures $6(\mathrm{a})$ and $6(\mathrm{~b})$ show the classification results without and with the ferry, respectively. While the SPN percentage does not change after the ferry is introduced, a significant percentage of A-DTN classification is converted to U-DTN classification, demonstrating the benefits of introducing the ferry.

Now also consider the question of whether the ferry should be used exclusively for data transfer or used to provide additional contacts (in addition to existing node contacts). Figure $6(\mathrm{c})$ shows how the network classification changes when the ferry is used exclusively. When compared to figure $6(\mathrm{~b})$ one can see that the network connectivity is now through space-time paths only. The WAM continuum framework provides an intuitive approach to understand the difference between these two network routing approaches in the presence of ferries.

\section{Adaptive real-time network operation.}

It is also possible to use network classification as a guide to adapt network operation. What would be required in this case is a system that first develops a full picture of network classification as a function of network parameters. This would be an off-line exercise similar to the examples we have already shown in the paper. Next, one would need to implement an on-line classification system that would allow the network nodes to determine their current classification (i.e., position in the continuum). As the classification changes, the network would adapt its operation. For example, consider the ferry example above. a network with 50 nodes can initially operate without a ferry. According to figure 6(a) the network is well-connected and does not need a ferry. Over time if the number of nodes drops to 20 or below, the same figure shows that the network becomes mostly of the A-DTN type and in need of "assistance". The network may then proceed to deploy a ferry (which could have been a node on standby for such a purpose). Figure 6(b) shows that the introduction of a ferry will result in a network that is mostly U-DTN.

Perhaps the most challenging aspect of this scenario is the need for a distributed, on-line system that would allow network nodes to classify the network in a distributed and real-time manner. This is a challenging problem and is the subject of on-going research. Some preliminary thoughts on how this may be accomplished are documented in [16].

\section{Generalizing the framework into other dimensions.}

It is also possible to use the WAM continuum framework to provide other insights into network behavior beyond issues of connectivity. In [1] we use our framework to develop a classification of networks based on energy availability. With this framework we are able to provide an understanding of a WAM's energy "sufficiency" depending on a combination of network connectivity properties, available energy, and power management scheme.

\section{CONCLUDING REMARKS}

In this paper, we develop the WAM Continuum framework which aims to provide a unified treatment of wireless and mobile networks. The framework is based on the construction of a WAM continuum that defines the space of 
networks and a corresponding formalism by which one can group related WAMs into classes that map into design and operational regimes. We present a specific instantiation of this framework that classifies networks according to their path properties. We illustrate our approach by applying it to networks described by traces from both real deployments and mobility models. Our examples demonstrate the power of our framework to provide a systematic and formal way to reason about the diverse space of wireless and mobile networks in a uniform manner. We also discuss the potential of our framework as an evaluative and comparative tool for comparing network alternatives as well as understanding the effect on network design and parameters on a WAM's character and performance.

\section{REFERENCES}

[1] Y. Chen, V. Borrel, M. Ammar, E. Zegura, "A Framework for Characterizing the Wireless and Mobile Network Continuum (Extended Version)," Technical Report, School of Computer Science, Georgia Institute of Technology, 2009. http://www.cc.gatech.edu/ãmmar/WAMtechreport.pdf

[2] C. E. Perkins and E. M. Royer, "Ad hoc on-demand distance vector routing," in Proceedings of the 2nd IEEE Workshop on Mobile Computing Systems and Applications, 1999.

[3] D. B. Johnson, D. A. Maltz, and J. Broch, "DSR: The dynamic source routing protocol for multi-hop wireless ad hoc networks," in In Ad Hoc Networking. Addison-Wesley, 2001.

[4] A. Vahdat and D. Becker, "Epidemic routing for partially connected ad hoc networks," Duke University, Tech. Rep. CS-200006, 2000.

[5] T. Spyropoulos, K. Psounis, and C. S. Raghavendra, "Spray and wait: an efficient routing scheme for intermittently connected mobile networks," in Proceeding of the ACM WDTN, 2005.
[6] A. Lindgren, A. Doria, and O. Schelen, "Probabilistic routing in intermittently connected networks," in Proc. of the SAPIR, 2004.

[7] S. Merugu, M. Ammar, and E. Zegura, "Space-time routing in wireless networks with preictable mobility," College of Computing, Georgia Tech, Tech. Rep. GIT-CC-04-07, 2004.

[8] V. Borrel, M. Ammar, and E. Zegura, "Understanding the wireless and mobile network space: A routing-centered classification," in Proceedings of CHANTS, 2007.

[9] P. Jacquet, "Space-time information propagation in mobile ad hoc wireless networks," in IEEE Information Theory Workshop, 2004.

[10] M. Grossglauser and M. Vetterli, "Space-time routing in ad hoc networks," in In Proc. Ad Hoc Now, 2003.

[11] A. Ferreira, "Building a reference combinatorial model for MANETs," IEEE Network, vol. 18, no. 5, 2004.

[12] J. Scott, P. Hui, J. Crowcroft, and C. Diot, "Haggle: A networking architecture designed around mobile users," in proceedings of IFIP WONS, 2006.

[13] J. Scott, R. Gass, J. Crowcroft, P. Hui, C. Diot, and A. Chaintreau, "CRAWDAD trace cambridge/haggle/imote/intel (v. 2006-01-31)."

[14] A. Keränen, J. Ott, and T. Kärkkäinen, "The one simulator for dtn protocol evaluation," in SIMUTools '09: Proceeding of the 2nd International Conference on Simulation Tools and Techniques, 2009.

[15] W. Zhao, M. Ammar, and E. Zegura, "A message ferrying approach for data delivery in sparse mobile ad hoc networks," in Proc. of ACM MobiHoc, 2004.

[16] D. Antonellis, A. Mansy, K. Psounis, and M. Ammar, "Towards Distributed Network Classification for Mobile Ad Hoc Networks," in Proceedings of the Wireless Internet Conference (WICON), 2008. 